Meta

Journal des traducteurs

Translators' Journal

\title{
Traduire les réseaux métaphoriques chez Nuruddin Farah
}

\section{Laurence Jay-Rayon}

Volume 52, numéro 4, décembre 2007

La traduction et les études de réseaux

Translation and Network Studies

URI : https://id.erudit.org/iderudit/017699ar

DOI : https://doi.org/10.7202/017699ar

Aller au sommaire du numéro

Éditeur(s)

Les Presses de l'Université de Montréal

ISSN

0026-0452 (imprimé)

1492-1421 (numérique)

Découvrir la revue

Citer cet article

Jay-Rayon, L. (2007). Traduire les réseaux métaphoriques chez Nuruddin Farah. Meta, 52(4), 839-858. https://doi.org/10.7202/017699ar

\section{Résumé de l'article}

Cet article analyse les enjeux de la traduction de réseaux métaphoriques dans le roman Sardines de l'auteur somalien d'expression anglaise Nuruddin Farah. Il tente dans un premier temps de mettre en évidence la fonction des réseaux métaphoriques à l'échelle du roman original puis, dans un second temps, d'explorer les enjeux de leur traduction en proposant des pistes de réflexion. Au-delà de cet objectif, cette étude s'efforce de mettre en évidence d'autres types de réseaux sous-tendant l'écriture de Farah et que le traducteur littéraire peut difficilement ignorer s'il veut rendre un juste hommage aux réalités linguistiques, poétiques et socioculturelles auxquelles l'auteur a puisé. Pour ce faire, cet article s'appuie notamment sur les approches théoriques des traductologues Barbara Folkart et Antoine Berman, et ouvrira sur des suggestions plus générales pour la traduction de réseaux en littérature.
Ce document est protégé par la loi sur le droit d'auteur. L'utilisation des services d’Érudit (y compris la reproduction) est assujettie à sa politique d'utilisation que vous pouvez consulter en ligne.

https://apropos.erudit.org/fr/usagers/politique-dutilisation/ 


\title{
Traduire les réseaux métaphoriques chez Nuruddin Farah
}

\author{
LAURENCE JAY-RAYON \\ Université de Montréal, Montréal, Canada \\ laurence.ibrahimaibo@gmail.com
}

\begin{abstract}
RÉSUMÉ
Cet article analyse les enjeux de la traduction de réseaux métaphoriques dans le roman Sardines de l'auteur somalien d'expression anglaise Nuruddin Farah. II tente dans un premier temps de mettre en évidence la fonction des réseaux métaphoriques à l'échelle du roman original puis, dans un second temps, d'explorer les enjeux de leur traduction en proposant des pistes de réflexion. Au-delà de cet objectif, cette étude s'efforce de mettre en évidence d'autres types de réseaux sous-tendant l'écriture de Farah et que le traducteur littéraire peut difficilement ignorer s'il veut rendre un juste hommage aux réalités linguistiques, poétiques et socioculturelles auxquelles l'auteur a puisé. Pour ce faire, cet article s'appuie notamment sur les approches théoriques des traductologues Barbara Folkart et Antoine Berman, et ouvrira sur des suggestions plus générales pour la traduction de réseaux en littérature.
\end{abstract}

\begin{abstract}
This paper aims to examine the challenges at stake when translating metaphor networks in Sardines, a novel by the English-speaking Somali writer Nuruddin Farah. Its purpose is, on one hand, to underline the function of metaphor networks within the original novel, and, on the other hand, to explore the challenge of their translation and to give some thought to it. Beyond this aim, this work will attempt to reveal links with other types of networks within Farah's writing that the literary translator willing to pay tribute to the East-African linguistic, poetic, social and cultural realities that inspired the novel can hardly ignore. To do so, this paper relies on the concepts developed by - among others - translation studies scholars Antoine Berman and Barbara Folkart, and will suggest new avenues on a broader scale for translating networks in literature.
\end{abstract}

\section{MOTS-CLÉS/KEYWORDS}

oralité, Afrique de l'Est, postcolonialisme, poésie, altérité

\section{Introduction}

Cet article fait suite à une recherche de plus grande ampleur réalisée dans le cadre d'un mémoire de maîtrise (Jay-Rayon 2006) et dont l'objectif était de souligner les enjeux de la traduction de métaphores dans le roman d'expression anglaise Sardines de l'auteur somalien Nuruddin Farah. Le corpus ayant servi de support à cet article et au mémoire était composé du texte original anglais de Nuruddin Farah, Sardines (1992), et de sa traduction française réalisée par Christian Surber, Sardines (1995). Pour constituer à proprement parler des réseaux métaphoriques, les métaphores relevées dans Sardines devaient à la fois s'inscrire dans l'une des thématiques récurrentes du roman incarnant les préoccupations de Farah et être liées entre elles par l'un des réseaux signifiants sous-jacents traversant le texte. Les fondements théoriques de cet article reposant largement sur les concepts bermaniens, notamment sur 
l'importance à accorder à la lettre du texte source, la définition des réseaux signifiants sous-jacents retenue dans le cadre de cet article est celle établie par Berman en 1985. Dans la première partie, je fais un rapide tour d'horizon de la métaphore et des difficultés liées à sa traduction dans différents domaines (général, publicitaire, scientifique) avant d'aborder la spécificité du littéraire en présentant certains des concepts proposés par Berman et Folkart et d'introduire les notions de dialogisme et d'intertextualité établis par Bakhtine. La seconde partie présente l'auteur Nuruddin Farah et son œuvre puis aborde la question de la littérature orale somalie et des caractéristiques formelles de la poésie somalie. La troisième et dernière partie montre en quoi la création métaphorique est caractéristique des narrations de Farah puis présente l'étude de cas à proprement parler.

\section{Les «réseaux métaphoriques» et leur traduction : jalons théoriques}

La traduction de réseaux métaphoriques pose un véritable défi au traducteur de textes tant à visée communicative que littéraires. Si, selon la propre définition d'Aristote, la métaphore correspond au transport à une chose d'un nom qui en désigne un autre, ou au transport du genre à l'espèce, ou de l'espèce au genre, ou encore de l'espèce à l'espèce (Tamba-Mecz 1981: 41), la métaphore a depuis inspiré bon nombre de linguistes, de cognitivistes, d'éthiciens ou de philosophes dont les réflexions ont permis de dépasser le simple cadre de la figure de style. Les travaux des cognitivistes Lakoff, Johnson et Turner proposent notamment d'aborder la métaphore sous l'angle conceptuel, ce qui correspond, comme le formule Sylvie Vandaele, à «l'établissement de correspondances entre un domaine-source et un domaine-cible» (Vandaele 2002: 224). Selon Lakoff et ses collaborateurs, la métaphore n'est donc pas une figure rhétorique ornementale, mais - au contraire - un mécanisme fondamental de pensée et d'action: « $[\mathrm{M}]$ etaphor allows us to understand our selves and our world in ways no other modes of thought can» (Lakoff et Turner 1989: xi): «[M] etaphor is anything but peripheral to the life of the mind. It is central to understanding our selves, our culture, and the world at large» (Lakoff et Turner 1989: 214). La possibilité de création métaphorique repose sur l'existence de réseaux sémantiques. Les réseaux sémantiques constituent une représentation de l'organisation des connaissances en mémoire, ils permettent de relier entre eux des éléments significatifs qui y sont stockés. Schématiquement, les concepts sont généralement représentés par des nœuds et les relations entre les concepts, par des arcs. Sandra Halverson, s'appuyant sur la théorie de la grammaire cognitive de Langacker (1987), résume la structure sémantique de la manière suivante:

[...] semantic structure may be characterized in terms of integrated networks. These networks are complex and dynamic and are accessed by symbolic units (bipolar structures). There are various kinds of knowledge included in these networks, including perceptual knowledge, extralinguistic "real-world" knowledge and metalinguistic information. (Halverson 2003: 209)

Par bipolarité, Halverson et Langacker entendent l'existence de deux pôles: sémantique et phonologique. Je reviendrai plus tard sur cette notion de bipolarité, car celle-ci s'avérera utile lors du processus d'analyse des exemples présentés. L'illustration 
suivante donnée par Halverson introduit d'ailleurs l'un des mécanismes sur lesquels repose l'appareil métaphorique de Farah:

[...] a network for aunt, for example, may include extralinguistic information, such as the existence of alternative phonological variants /ants/vs./ænt/ and relevant information concerning them. (Langacker 1987, cité par Halverson 2003: 206)

Je propose de retenir la même représentation schématique que celle qui est communément utilisée et retenue pour les réseaux sémantiques, à savoir la notion de concept et de liens les unissant. C'est sur cette schématisation que je m’appuierai, ainsi que sur la notion de bipolarité - sémantique et phonologique - pour analyser les réseaux métaphoriques et leur traduction. À partir de cette schématisation, je définis la notion de réseau métaphorique comme l'ensemble des métaphorisations unies par une relation (formelle, lexico-sémantique ou autre) au sein d'un thème donné.

Comme le soulignent Lakoff et ses collaborateurs, la pratique métaphorique est intimement liée au système conceptuel de chaque société et de chaque culture. Anton Rydning évoque d'ailleurs la difficulté que pose la traduction de tropes tels que métaphores et métonymies, puisque notions et situations sont généralement exprimées de manière différente selon les langages-cultures en question:

Les différentes langues ne choisissent pas les mêmes aspects pour désigner les mêmes concepts. Il suffit de se reporter aux adages dans deux langues différentes pour constater qu'ils désignent le même concept au moyen de mots différents. Ainsi, porter de l'eau au moulin correspond en anglais à to bring coals to Newcastle. Bien que les images qu'évoquent ces deux expressions soient différentes, elles renvoient toutes deux au fait de donner involontairement à quelqu'un des arguments dans un débat. (Rydning 2004: 859)

Cet exemple souligne le fait qu'un même concept puisse renvoyer à différentes réalisations linguistiques. Selon Lakoff (1987) cependant, nos systèmes conceptuels notamment le travail de catégorisation à la base de ces mêmes systèmes - seraient étroitement liés à notre culture et à notre système linguistique. D’après cette théorie, les concepts sous-jacents à une métaphore exprimée, par exemple, par un nonOccidental, pour prendre l'ensemble le plus large qui soit, n'auraient ainsi pas nécessairement d'équivalents pertinents dans le monde conceptuel occidental. Dans le cas de Farah, il convient sans doute d'apporter quelques précisions. En effet, si Nuruddin Farah est de culture est-africaine - somalienne pour être plus précise - il a été scolarisé en anglais dès son plus jeune âge et a été imprégné de littérature écrite anglophone, amharique et arabe, tout en baignant parallèlement dans un univers de littérature orale traditionnelle somalie. On pourrait donc qualifier ses influences culturelles (et par conséquent conceptuelles) d'hybrides, ce qui - selon les constatations mêmes de Lakoff (1987: 84) - exclurait Farah d'un système de catégorisation "purement somali». Cependant, il est évident que l'analyse - par un traducteur - de métaphores conçues par un auteur de culture non occidentale et exprimées avec un matériau linguistique dissocié de cette culture sous-entend une confrontation d'autant plus complexe, supposant un travail d'analyse minutieux ainsi qu'une recherche exégétique poussée. Michele Prandi souligne d'ailleurs le rôle de l'«interprète» de métaphores en ces termes:

En l'absence de rails préétablis, le travail d'interprétation de la métaphore confère à l'interprète un espace virtuellement ouvert, et par conséquent met en relief sa respon- 
sabilité, exactement comme l'interprétation non littérale d'énoncés cohérents. (Prandi 2000: 26)

À propos de la traduction scientifique et technique, en langues de spécialité, Stambuk souligne l'importance de l'analyse des concepts et des liens qui les unissent:

[...] en effet, la conceptualisation abstraite nécessite l'appropriation de liens déjà établis dans des domaines concrets et directement accessibles à l'expérience, ce qui en retour facilite l'appréhension de nouveaux concepts (Stambuk 1998, cité par Vandaele 2002: 225).

Ainsi, selon S. Vandaele:

Une question fondamentale en traduction devient alors le passage du système conceptuel auquel a recours la langue de départ à celui de la langue d'arrivée. Autrement dit, pour un domaine particulier, y a-t-il, ou n'y a-t-il pas, correspondance entre les métaphores conceptuelles véhiculées par les langues en présence? Quelles en sont les conséquences sur la prise de décision traductionnelle, tant en ce qui a trait au terme qu'à la phraséologie? Se poser ces questions revient d'emblée à s'affranchir de la recherche étroite de l'équivalence lexicale, pour prendre en compte les connaissances de nature encyclopédique sous l'angle de leur organisation conceptuelle. (Vandaele 2002: 225)

Dans le domaine publicitaire, Christian Papas (2007) présente un exemple de métaphore illustrant la difficulté de rendre en français une métaphore en grec reposant sur un jeu de mots sur la lexie «rame». La publicité met en scène deux journalistes en vacances dans une barque de pêche et recevant un coup de téléphone de leur patron leur demandant de rentrer travailler; le premier demande alors au second de prendre la/les rames et ce dernier lui répond "pour une rame d'information» (traduction mot à mot du grec). Cette métaphore vive ne peut malheureusement se traduire telle quelle en français "puisque "rame" n'est colloqué avec rien» (Papas 2007). Papas propose de recourir à la métaphore lexicalisée en français «des informations à la pelle» ce qui, malheureusement, annihile l'allusion au contexte (barque, pêche, rame). Opter pour cette solution gomme le lien avec le réseau iconographique (le paratexte) qui, en publicité notamment, joue un rôle majeur. Papas avance pour sa part le parallélisme lexico-sémantique entre rame et pelle, mais - le principe de la publicité reposant sur la rapidité des connexions à établir - il me semble audacieux de compter sur la capacité du lecteur à faire le lien dans les délais requis.

Il convient à présent de s'interroger sur les enjeux propres au domaine littéraire, bien que l'on puisse avancer la théorie selon laquelle ces enjeux ne constituent pas l'apanage de ce seul domaine. Je pose cependant que la traduction de réseaux métaphoriques en littérature constitue un défi supplémentaire pour leur interprète (traducteur) dans la mesure où ils s'inscrivent dans un ensemble de réseaux multiples formant un "tout-texte». Louise Audet, dans sa thèse de doctorat, cite Molinié (1993: 15) qui définit le texte comme le résultat de relations organisant des «faits verbaux» :

[...] le texte est le résultat d'un tissu de relations, de tous ordres, qui elles seules constituent, par leurs réseaux mutuels, le tout global - ce qu'on appelle exactement une structure. Ces relations organisent évidemment des faits verbaux: thématiques, dramatiques, narratifs, syntaxiques, lexicaux, figurés, énonciatifs, rythmiques, sonores; ces relations se tissent entre ces divers niveaux ou composantes, et entre chacune d'elles et le tout-textuel. (Molinié, cité par Audet 2006) 
La difficulté particulière de la traduction de métaphores en contexte littéraire tient ainsi à son inscription au sein de multiples réseaux, et donc à la multiplicité des relations possibles. Je soulignerai au cours de l'étude de cas la présence de réseaux autres que purement métaphoriques - réseaux ne relevant pas de la sémantique - que l'analyse de la traduction des métaphores de Sardines a permis de mettre en lumière et qui caractérisent les tropes de la narration de Farah. Jeanne Dancette a établi dans ses travaux sur les processus cognitifs en traduction que le traducteur travaillait simultanément sur cinq axes, en établissant le modèle de la double hélice qui «représente l'interaction entre les textes, les langues et les connaissances, dans un (ou plusieurs) univers de discours» (Dancette et al. 2007: 115). Ces cinq axes (intraréférentiel, formel, sémantique, narratif et traductologique) peuvent constituer une grille intéressante pour repérer les réseaux signifiants sous-jacents du texte littéraire.

La créativité en traduction littéraire présente des spécificités. L’axe formel y revêt une plus grande importance que dans la traduction de communication: l'axe référentiel se renforce par l'axe intraréférentiel (Folkart 1991; Meschonnic 1999); et l'axe narratif - généralement absent (ou très peu développé) dans la traduction de communication - s'ajoute. (Dancette et al. 2007: 118)

Quels sont les autres apports traductologiques susceptibles d'aider le traducteur littéraire à prendre conscience de la notion de réseau en traduction littéraire? Indiscutablement, la théorie bermanienne offre une série d'outils très précieux. En effet, Antoine Berman propose comme préalable à la traduction littéraire une analyse fouillée du texte de départ, répertoriant par la même occasion une série de tendances déformantes propres au traducteur (Berman 1995). La non-reconnaissance des réseaux signifiants sous-jacents s'inscrit dans cette série de treize tendances déformantes et constitue un élément extrêmement pertinent dans le cadre de cette étude, aux côtés de la destruction des rythmes, de l'appauvrissement qualitatif et de la destruction des locutions (Berman 1995) qui sont des critères auxquels le traducteur confronté aux métaphores de Sardines doit prêter une attention toute particulière. Avant d'aller plus loin, je propose de m'attarder sur la définition des « réseaux signifiants sous-jacents » au sens bermanien, puisque c'est sur cette définition-là que je me suis appuyée tout au long de cette recherche. Berman précise ainsi que:

Toute œuvre comporte un texte «sous-jacent», où certains signifiants-clefs se répondent et s'enchaînent, forment des réseaux sous la «surface» du texte, je veux dire: du texte manifeste, donné à la simple lecture. C'est le sous-texte, qui constitue l'une des faces de la rythmique et de la signifiance de l'œuvre. (Berman 1999: 61)

Pour illustrer son propos, Berman cite notamment l'utilisation des augmentatifs chez Arlt, qui - alors que disséminés dans l'œuvre et donc non repérables comme système à la première lecture - une fois mis en réseau, constituent un enchaînement cohérent et même «l'une des dimensions essentielles des Sept Fous» (Berman 1999: 62). Je rejoins Berman lorsqu'il affirme: "La traduction qui ne transmet pas de tels réseaux détruit l'un des tissus signifiants de l'œuvre.» (Berman 1999: 62). La notion bermanienne de réseaux sous-entend donc le caractère non ostentatoire des éléments formant des réseaux à l'échelle d'une œuvre. À ses yeux, c'est le repérage d'une systématique qui permet de mettre des éléments bout à bout et de leur donner une cohérence. Ces éléments peuvent tenir aussi bien du formel que du sémantique, l'un pouvant d'ailleurs contribuer à la mise en réseau de l'autre: pour revenir sur l'exemple des 
Sept Fous, le repérage des augmentatifs en -òn prend valeur de décodage d'un réseau de signifiance supérieure, qui représente l'une des dimensions essentielles de l'œuvre (Berman 1999: 62). La notion bermanienne de signifiance n'est d'ailleurs pas sans rappeler la distinction entre sens et signifiance établie par Riffaterre «lorsqu'il parle d'un syntagme donné, qui n'atteint pleinement sa signifiance qu'une fois que se réalise le lien intertextuel - jusqu'alors demeuré incomplet» (Charron 2001: 117).

À l'instar de Berman, je me suis attardée sur la valeur et la signifiance de la lettre lors de cette étude de cas. En effet, les tropes élaborés dans la narration de Farah présentent la particularité d'être investis de deux valeurs fondamentales, autant formelle que sémantique. Je souligne que Berman s'est intéressé au "polyfacétisme», c'est-à-dire à «ce tissu signifiant que constituerait le mélange de l'oral et de l'écrit » du roman latino-américain (Charron 2001: 99). Les termes de "polyfacétisme» et «d'espace polylangagier» rappellent d'ailleurs en tous points la terminologie de Bakhtine; et il est à ce propos intéressant de souligner comment Berman kidnappe le concept de dialogisme introduit par Bakhtine (relations d'intertextualité et d'intratextualité, qui constituent d'ailleurs l'une des formes de réseaux signifiants sousjacents, comme nous le verrons dans l'étude de cas) pour asseoir sa position éthique: "établir un rapport dialogique entre langue étrangère et langue propre» (Berman 1985, cité par Charron 2001 : 101). Selon Berman, il est primordial de s'attacher à la lettre et à l'aspect formel du texte source pour faire place à l'altérité du texte de départ dans le texte d'arrivée: à ses yeux, les choix lexicaux dans le texte source sont investis d'une valeur s'inscrivant bien au-delà de la simple fonction cognitive, véhiculant une poétique, un rythme, une musique. C'est également sur cette dualité entre le fond et la forme que s'est penchée Barbara Folkart qui souligne cependant, bien qu'en proposant une grille d'analyse différente, le non-parallélisme entre processus d'écriture originale et processus traductif: " the thinking translator does as (not what) the source-language author did»(Folkart 1999: 89). Du Conflit des énonciations de Folkart, j'ai ainsi relevé tout particulièrement le concept de voix de l'auteur, les notions de filtrage (saisie des référents, du syntagme, des contenus pragmatiques et des récurrences), de décalage traductionnel et de remédiation (Folkart 1991).

Avant de passer à la présentation commentée des exemples, il est essentiel de s'attarder sur les contextes qui ont entouré l'écriture de la fiction étudiée dans la mesure où l'extratextuel joue un rôle majeur dans le travail d'analyse qui suit.

\section{Farah, à la croisée de plusieurs styles littéraires}

Farah fait partie des écrivains africains anglophones postcoloniaux, à l'instar de compatriotes tels que le Nigérian Chinua Achebe ou le Kenyan Ngũgĩ Wa Thiong’o, dont il se distingue cependant, dépassant le discours de l'identité et du nationalisme culturels pour créer son propre univers référentiel, n'hésitant pas à s'inspirer aussi bien de Virginia Woolf, de Franz Kafka ou de James Joyce que du Nigérian Wole Soyinka (Gikandi 1998). L'unicité de l'écriture de Farah réside en sa capacité à explorer les possibilités offertes à la fois par la tradition orale de la poésie somalie et par les courants littéraires contemporains. Farah prend par exemple ses distances avec les prises de position de Ngũgĩ voyant le médium anglais comme une «bombe culturelle» (Ngũgĩ 1986) pour se rallier implicitement au point de vue de Salman Rushdie, qui considère au contraire que c'est l'appropriation même du médium linguistique de 
l'ex-colonisateur qui permettra aux anciens colonisés de clore le processus de libération: "And I hope all of us share the view that we can't simply use the language in the way the British did; that it needs remaking for our own purposes» (Rushdie 1992: 17).

L'importance de la littérature orale en Somalie, notamment de la poésie, est primordiale. Comme le soulignent à juste titre Andrzejewski et Lewis: «It is perhaps not too much to claim that the Somalis are a nation of bards: and their poetry certainly is one of their principal cultural achievements» (Andrzejewski et Lewis 1964: 3). Langue orale comme bon nombre de langues africaines, le somali n'a obtenu un statut de langue écrite qu'en 1972. Le patrimoine poétique somali dans lequel Farah - né en 1945 - a baigné est de toute évidence marqué par l'oralité. Cette domination de la langue orale s'est trouvée renforcée par son environnement familial, sa mère composant poésies et chansons orales et son père étant interprète pour le gouverneur britannique de l'époque. Cette caractéristique n'est d'ailleurs pas l'apanage de la littérature somalie, comme le Nigérian Herbert Igboanusi le souligne:

The fusion of the oral tradition in the written medium forms the core of the distinctness of the Igbo novel. [...] In this way, written literature complements oral literature rather than being a substitute of it. (Igboanusi 2002: 9).

Les poèmes somalis sont en général dédiés à une thématique sociale ou politique plutôt qu'à une préoccupation individuelle. Ils peuvent être utilisés comme instrument de lobbysme ou pour illustrer une problématique sociale, politique ou religieuse du moment. Au-delà de sa valeur de joute rhétorique très prisée par les nomades, la poésie somalie est caractérisée par une pratique «rhétorique» qu'il convient de souligner en ce sens qu'elle se rapproche du processus métaphorique; je veux parler ici $\mathrm{du}$ «sarbeeb» que l'universitaire somalien Said Samatar traduit par « the art of oblique communication» (Samatar 2005) en soulignant que: "Among the Somalis of the Horn of Africa, the dominant medium for addressing a hidden discourse is poetry - oral and written.» (Samatar 2005: 1 [Editor's note]). Samatar donne ainsi l'exemple d'un poème mettant en scène une jument sachant à peine galoper pour suggérer indirectement une jeune épouse ne sachant pas tenir son intérieur. Le paysage poétique somali ainsi campé, on comprend sans peine la visée didactique de tropes tels que les métaphores pour transmettre indirectement un message.

J'aimerais d'autre part souligner l'une des caractéristiques formelles de la poésie somalie, car celle-ci jouera un rôle majeur dans le cadre de cette analyse: l'emploi systématique et marqué des allitérations (higaad, en somali). Leur utilisation, associée à celle de l'argumentation indirecte, rend parfois complexe l'interprétation de poèmes, ceux-ci demandant une excellente connaissance de la motivation de l'auteur et des contextes entourant leur écriture. Je montrerai plus loin à quel point cet aspect formel s'exprime à travers les réalisations métaphoriques dans le texte de Farah. Je n'entrerai pas dans le détail de la complexité métrique, autre caractéristique formelle de la poésie traditionnelle somalie, dans la mesure où celle-ci n'est pas pertinente dans le cadre de cette recherche. Par contre, il est intéressant de signaler l'existence d'une tradition poétique somalie s'appuyant sur le concept de réseaux; le terme silsilad, en somali, désigne une série de textes formant des réseaux, une chaîne constituée des réponses des membres d'un groupe à un adversaire commun (Morin 1997: 5). Il est notamment intéressant de souligner qu'à l'époque où Farah écrivait Sardines, 
le poète somali Maxamed Xaashi Dhamac «Gaarriye» avait quant à lui recours aux «poetic chains ${ }^{1} »$ pour protester contre la dictature de Siyaad Barre. Ce mode d'expression poétique contestataire consistait à lancer une première diatribe poétique à laquelle répondait une autre personne, le tout formant ensuite une véritable chaîne. Dans une entrevue accordée au Guardian, Gaarriye précise:

One poet starts a theme, others take it up, then a chain develops, drawing people into the arguments. I wrote something against tribalism that became an attack on the president. [...] Within four months the chain was more than 70 poems long. [...] And that was when the secret police came to visit. (Maxamed Xaashi Dhamac «Gaarriye» cité par Rushby 2005: 1)

Certaines de ces chaînes poétiques, notamment celles de Gaarriye, devaient se conformer à une règle formelle: respecter des allitérations convenues au départ. On pouvait par exemple retrouver des chaînes de poésies obéissant à un mode allitératif en $D$. La poésie somalie, tout orale qu'elle soit, répond donc à un système de classification complexe assorti d'une hiérarchie très stricte, et si mon propos n'est pas ici d'en présenter la complexité, il convient d'en connaître l'existence avant de se livrer au travail exégétique que requiert l'analyse des réseaux métaphoriques de Farah afin de pouvoir établir les liens nécessaires avec des pratiques orales reconnues.

\section{Les réseaux métaphoriques à l'œuvre dans Sardines}

Quatre principaux réseaux sémantiques se dégageaient du corpus analysé (Sardines et sa traduction) : pouvoir (assortis de deux sous-thèmes, à savoir caractères/personnalités et oppression/violence); liberté/épanouissement; plaisir; fertilité/naissance. Les exemples présentés ici ont été respectivement puisés dans les première (pouvoir/ oppression/violence) et dernière (fertilité/naissance) catégories. Je rappelle que pour répondre à la définition de réseaux métaphoriques, les métaphores relevées dans le corpus devaient à la fois s'inscrire dans l'une des quatre thématiques du roman et être liées entre elles par l'un des réseaux signifiants sous-jacents traversant le texte.

Abdourahman Ali Waberi, à propos de la création métaphorique dans les narrations de Farah, s'exclame:

The reader is endlessly impressed by a perpetual metaphorization of everything that stems from abstraction, to such an extent that one could establish a physiognomy of Nuruddin Farah's fiction. This physiognomy, which some would call the narrative's physique, strikes me as having a double function: it is at once a means of knowledge and a narrative form. (Waberi 1998: 775)

Waberi souligne par ailleurs que la pratique métaphorique (notamment celle de la métaphore organique qui faisait l'objet de son article) est loin d'être l'apanage de Farah, mais qu'elle est au contraire pratique courante chez bon nombre d'écrivains africains (nord-africains y compris). Il précise cependant que celle de Farah se rapproche de la poésie somalie, en cela que toutes deux cultivent l'ambiguité: «In the case that interests us here, Farah, like any good Somali poet, plays on the ambiguities of the esthetic units» (Waberi 1998: 778), une affirmation que confirme Johnson: "An image can carry a double or hidden message... This device is common enough in Somali poetry» (Johnson 1974, cité par Waberi 1998: 778).

Sardines constitue le second roman de la première trilogie de Nuruddin Farah, intitulée Variations on the Theme of an African Dictatorship, et dont la dictature de 
Siyaad Barre représente la toile de fond. Par le biais de métaphores, Farah illustre les principaux fléaux ayant marqué cette époque: culte de la personnalité, manque d'instruction, clanisme (que Farah qualifie de pratique incestueuse) et dictature sous toutes ses formes (intolérance, déportations, exécutions) et dans tous les contextes de la vie quotidienne personnelle (familiale, sociale) et professionnelle.

Pour ce faire, Farah met en scène une majorité de personnages féminins: Médina - la principale protagoniste - femme moderne et affranchie du joug de la religion et du clanisme, qui vient à la fois de perdre son poste de rédactrice en chef pour avoir osé critiquer le gouvernement en place et de se séparer de son mari Samater, récemment nommé ministre des Constructions; la mère de Samater, Idil, femme tyrannique qui entend régir la vie de son fils et de sa belle-fille et menace d'exciser leur petite fille de huit ans, Ubax. Parallèlement à cette intrigue principale, Farah met en scène des personnages secondaires dont les itinéraires viennent illustrer les thématiques féministes dont Farah a fait son cheval de bataille: le droit à l'éducation et à la santé, la liberté (politique, sociale, sexuelle) par opposition à la violence (oppression, esclavage social et familial, viol). On découvre notamment les personnages de Sagal (athlète qui papillonne entre différentes causes et peine à mener un projet à terme), de Dulman (chanteuse analphabète attendant en vain de pouvoir concevoir un enfant), d'Amina (fille d'un ministre du gouvernement de S. Barre, violée par un groupe d'hommes voulant se venger de son père) et de Fatima bint Thabit (mère de Nasser et Médina, figure féminine vivant dans l'ombre et la soumission). Les personnages masculins illustrent toute une gamme de profils, du plus autoritaire et tyrannique (le grand-père maternel de Médina) jusqu'au plus faible (Samater, mari de Médina), en passant par des personnages intermédiaires comme Nasser (frère de Médina, complice des femmes et de leur combat).

Le traducteur de Sardines, Christian Surber, a traduit l'ensemble de la trilogie de Variations autour du thème d'une dictature africaine, à commencer par Du lait aigredoux (Sweet and Sour Milk) en 1994, puis Sardines en 1995 pour finir avec Sésame ferme-toi (Close Sesame) en 1997. Il a également traduit plusieurs romans d'auteurs africains et guyanais, et ses traductions sont en majorité publiées chez Zoé, une maison d'édition suisse.

Sur le plan méthodologique, voici quelques-uns des principaux critères de sélection des métaphores utilisés dans le cette recherche:

- le contexte (dans le sens large, à l'échelle du roman tout entier) joue un rôle essentiel pour interpréter les métaphores: c'est l'interaction entre les énoncés métaphoriques et la narration qui permet d'identifier les différents réseaux thématiques à l'œuvre;

- une place prépondérante est accordée à l'ancrage culturel des connotations évoquées par le référent et par le référé et à la pertinence des analogies établies entre ces deux éléments dans la traduction;

- les métaphores peuvent illustrer un concept original (the sun raged like an old man denied his rights) ou non ("la mort est un départ»), mais doivent utiliser un matériau linguistique original (Samater was weak in the head as he was in the knees), excluant par conséquent les métaphores lexicalisées ou "mortes" (it's raining cats and dogs);

- les éléments à l'œuvre dans l'énoncé doivent rapprocher deux réalités hétérogènes (My daughter is faceless like a windmill); 
- l'association entre ces deux réalités hétérogènes crée une image fusionnée (My daughter is faceless like a windmill and spins fast);

- cette image fusionnée doit non seulement tenir compte des connotations du référent, mais aussi de celles évoquées par le référé - produisant une interaction bidirectionnelle - (Ebla's smile was light like the water of childbirth);

- les énoncés établissant une similitude entre réalités hétérogènes à l'aide de la conjonction comme seront considérés ici comme métaphores (Sagal was fretful like a fish in agited water; Its passages flowed in her mouth like melted gold).

Les passages métaphoriques présentés ci-après ont été sélectionnés, outre du fait de leur appartenance à un réseau métaphorique majeur, en fonction des difficultés de balisage qu'ils représentaient pour le traducteur. Les différents réseaux à l'œuvre seront mis en évidence par une analyse aussi bien de la microstructure que de la macrostructure du texte source, et ce, en faisant appel aux concepts et aux outils proposés par Berman et Folkart. Les extraits seront présentés dans l'ordre suivant: tout d'abord la version originale anglaise accompagnée du passage en contexte, puis la version traduite - accompagnées de leur pagination respective - et seront suivis d'une analyse commentée.

Sagal was fretful like a fish in agitated water, malleable as a piece of metal under prolonged heat. She would exude enthousiasm for someone or something, then, like a lamp whose wick had dried, the fire in her would extinguish itself and die completely. (p. 27)

Sagal était nerveuse comme un poisson dans une eau agitée, malléable comme un fragment de métal soumis à une chaleur prolongée. (p. 41)

Cet extrait constitue la première phrase du second chapitre, consacré aux personnages de Sagal - championne de natation et d'indécision - et d'Ebla, sa mère. Le champ sémantique de l'eau est fortement présent tout au long des chapitres qui traitent de Sagal et forment un véritable réseau sémantique à l'échelle du roman. Élément dynamique, fluide et insaisissable, l'eau symbolise le personnage de Sagal et reflète également sa jeunesse. Le portrait qu'en fait Farah est celui d'une personne pétulante, débordant d'enthousiasme, mais toujours indécise, difficile à cerner et très influençable. La traduction de Surber confère au personnage une nervosité assimilable, selon lui, à celle d'un poisson en eau agitée. L'association de la nervosité et du poisson semble, de prime abord, curieuse et peu idiomatique. En français, on pense plutôt à la nervosité du cheval. A priori, l'adjectif fretful est - pris au premier degré - négatif (irritable, grognon, agité). Ici, le contexte et le caractère du personnage nous orientent vers une interprétation légèrement différente, caractérisant plutôt une personne toujours en mouvement. Sur le plan des sonorités, on dénote des allitérations en $F$ et en $M$ et une rime interne entre heat et piece. En s'éloignant d'une traduction mimétique, l'une des avenues possibles pour refléter la fonction de la métaphore et pour recréer l'effet global du notionnel et de l'émotionnel (Henry 2003) pourrait prendre la forme suivante: "Sagal frétillait comme un poisson frayant dans un torrent, malléable comme un fragment de métal soumis à la flamme du forgeron " (allitérations en $F$ et en $M$, rimes en $E N$, inscription dans le réseau sémantique de la thématique). Rejoignant les propos de J. Henry, j'avancerai que - dans l'exemple présenté ci-dessus - il convenait précisément de relever l'ancrage de ce passage dans de multiples réseaux, non seulement - sur le plan cognitif - dans le réseau métaphorique du pouvoir et dans celui du champ sémantique de l'eau, mais aussi dans les réseaux formels de 
sonorités: allitérations en $F$ et en $M$. Les allitérations constituent en effet un phénomène caractéristique des métaphores de Farah et il n'est pas inutile de rappeler que leur présence fait écho à une pratique traditionnelle de la poésie somalie caractérisée par l'utilisation d'allitérations. Le non-rendu de cet aspect de la lettre, au sens bermanien du terme, dans la traduction revient donc non seulement à ignorer un réseau d'ordre formel (la dimension orale des métaphores de Sardines), mais également à nier l'inscription de cette caractéristique dans un réseau plus large que constitue la tradition poétique somalie. La traduction proposée par Surber illustre ainsi parfaitement deux des tendances déformantes mises en lumière par Berman, à savoir la non-reconnaissance des réseaux signifiants sous-jacents et la destruction des rythmes, ainsi que le défi posé par le problème de distance (ici, socioculturelle) souligné par Folkart. Ne pas reconnaître la présence de ces réseaux dans le texte source vient par conséquent gommer l'ancrage socioculturel de la pratique narrative de Farah.

La série de métaphores qui suit posait des difficultés davantage liées à la cohérence au sein d'un réseau donné.

My daughter hasn't the political conviction, courage and maturity to paint the morning with anti-government slogans. (p. 27)

But she will not, I repeat, she will not paint the morning with anti-government writings. (p. 28)

Ma fille n'a pas les convictions, le courage et la maturité politiques qu'il faut pour ravaler le lever du jour avec la peinture de slogans antigouvernementaux. (p. 42) Mais en aucun cas, je le répète, en aucun cas elle ne va repeindre le matin d'une couche d'écrits antigouvernementaux. (p. 42)

Dans ce passage, Ebla remet en cause le dernier projet de sa fille, Sagal: manifester son opposition au gouvernement de manière ostentatoire. Cette métaphore filée (paint the morning/dawn with anti-governement slogans/against the General) revient à de multiples reprises tout au long du roman, avec de petites variantes indiquées ci-dessus entre parenthèses. La métaphore ne révèle pas ce que pourrait signifier concrètement paint the morning, même si elle suggère une action réalisée en secret - sans doute à la faveur de la nuit - et qui ne serait révélée qu'au petit matin, à la lumière du jour. Sur le plan de la traduction, il convenait de maintenir ce niveau d'ambiguïté entourant la métaphore paint the morning. Le traducteur a opté - dans la première occurrence - pour une réalisation des plus pragmatiques, faisant écho à l'expression figée ravaler la façade; la valeur sémantique conférée au terme ravaler semble ainsi s'éloigner de l'intention de l'auteur sur le plan du sens global et gommer la poésie induite par paint the morning. La métaphore traduite est, de plus, alourdie par l'explicitation ostentatoire avec la peinture de (traduction explicitante: Berman 1999). Dans la seconde occurrence, Surber a traduit de manière différente la même métaphore originale (paint the morning). C'est là ce que l'on pourrait qualifier de destruction des systématismes (Berman 1999). Cette métaphore filée joue un rôle majeur d'un bout à l'autre du roman, d'une part en raison de sa récurrence, d'autre part parce qu'elle illustre l'une des thématiques centrales de Sardines: la résistance au totalitarisme. Il convenait donc de repérer son rôle de véhicule thématique formant réseau et de respecter une cohérence dans la traduction pour conserver l'inscription de ces passages dans le réseau intratextuel de la narration. En faisant le choix d'un matériau linguistique appartenant à un référent différent (peinture en bâtiment) et 
en ignorant la valeur de répétition de la métaphore, le traducteur contribue au brouillage des pistes.

Avec l'exemple suivant, Farah fait écho à une métaphore précédente (Idil had a knife with which to purify the little girl's ufta! [p. 11]; Idil avait un couteau pour purifier l'ufta de la fillette [p. 21]) qui illustre l'un des grands réseaux signifiants du roman: les mutilations génitales féminines (MGF), excision et infibulation étant traditionnellement pratiquées dans la Corne de l'Afrique.

And you didn't even see the mutilated version. (p. 32)

Et même la version tronquée, tu ne l'as pas vue. (p. 48)

Ce passage fait allusion, lors d'une conversation entre Sagal et sa mère, à un film révolutionnaire sud-américain qui sert de prétexte à Farah pour souligner de façon indirecte une réalité extratextuelle (les MGF). La traduction de Christian Surber, non mimétique ici, trahit un non-repérage de ce réseau signifiant majeur. On ne pouvait en effet se permettre ici de s'éloigner des lexies mutilated/mutilée. Tronquée, en l'occurrence, n'avait aucun pouvoir évocateur de la thématique dont il était ici question. Il s'agissait davantage de repérer des réseaux signifiants (intratextualité) que de porter attention à la valeur formelle de la métaphore.

L'importance de la rythmique et des allitérations se retrouve, dans une moindre mesure, dans la réalisation suivante qui exige par contre un effort de repérage particulier sur le plan lexical.

And her mother Fatima bint Thabit was there, veiled from the top of the head to the tip of her toes in the dark traditionnal lengths of purdah. (p. 57)

Et sa mère Fatima bint Thabit se trouva là, voilée du sommet de la tête à la pointe des orteils dans les sombres longueurs traditionnelles de la purdah. (p. 79-80)

Au cours d'un échange avec Sagal, Médina s'évade en pensée et évoque tout d'abord sa mère, puis le père de celle-ci, ce patriarche ultra autoritaire ("a monstrosity», "the cruellest man these women had ever known»), pour qui les femmes n'étaient ni plus ni moins qu'un objet de reproduction, devant obéir au doigt et à l'oeil à leur époux («a woman's god is her husband»). Les termes associés dans cette métaphore à la purdah ${ }^{2}$ : dark, length (couvrant tout le corps) et traditional introduisent le passage qui va suivre, à savoir la description de ce grand-père tyrannique et tant détesté, incarnant cette tradition que les principales protagonistes de Sardines rejettent. Cette métaphore autour de la purdah suggère en quelques mots tous les éléments symbolisant la condition féminine en Somalie. L'adaptation en français ne posait pas de problème majeur sur le plan sémantique et les qualificatifs de la purdah se laissaient transposer tels quels. Cependant, le texte original comportait une dimension phonologique, Farah jouant ici à la fois sur le rythme et les allitérations du syntagme from the top of the head to the tip of her toes rappelant un virelangue. La traduction française passe à côté de la fonction «ludique» de cet aspect de la métaphore. Et si veil est présenté ici sous un aspect négatif (carcan), dans l'occurrence ci-dessous Farah met en scène la même lexie, mais en la connotant positivement (protection).

What is more, you wouldn't want her to invade Ubax's rosy sleep, to prick the little girl's intactness and hurt her to the depth of her veiled soul. (p. 59)

Qui plus est, tu ne voudrais pas la voir violer le sommeil de rose d'Ubax, froisser l'intégrité de la fillette et la blesser au plus profond du secret de son âme. (p. 83) 
Cet exemple s'inscrit, dans la version originale anglaise, deux pages plus loin que la métaphore précédente. La conversation entre Sagal et Médina se poursuit et Sagal avance une supposition: Médina aurait quitté Samater pour éviter qu'Idil ne mette sa menace à exécution à propos d'Ubax (exciser la petite fille). C'est à ce moment-là que la métaphore intervient, faisant écho à une image précédemment évoquée (a knife with which to purify the little girl's ufta). À plusieurs reprises dans le roman, Ubax voit Idil en cauchemar, munie d'un couteau et s'approchant d'elle. La traduction de la première partie de la métaphore est très pertinente (violer le sommeil) car elle fait écho aux MGF. Plus loin, Surber a également penché en faveur d'intégrité pour intactness, un choix inspiré dans la mesure où l'on parle en français d'«intégrité sexuelle» (comme en anglais d'ailleurs, "sexual integrity»). Le choix de la lexie froisser vient par contre brouiller la piste de l'image évoquée ci-dessus (intégrité sexuelle) et ne peut en aucun cas évoquer les MGF; or l'image ne peut être ambiguë ici: il s'agissait de reconnaître la gravité de la réalité extratextuelle, c'est-à-dire le référent pragmatique (Folkart 1991) pour faire le choix d'un matériau linguistique approprié (crever, déchirer, percer...). De plus, le choix de prick n'était pas anodin, si l'on considère que - comme le soulignent Waberi et Johnson - Farah cultive l'ambiguïté. Si le verbe prick signifie "piquer, crever» (le deuxième sens étant ici le plus pertinent), le substantif prick peut également se traduire - entre autres - par «con» (le sexe de la femme, en langage vulgaire). La traduction de ce syntagme passe ainsi complètement à côté de l'élément central de cette image. Dans la seconde partie de la métaphore (hurt her to the depth of her veiled soul), il s'agissait de reconnaître la dualité mise systématiquement en ouvre tout au long de la narration et qui constitue un réseau intrathématique. Le voile s'interprète ici comme une douce protection (voile protecteur), un abri, l'idée de la douceur du sommeil étant encore accentuée par la présence de rosy venant renforcer le sentiment de positivité suggéré par le voile (rosy était ici à interpréter au sens d'optimiste, idyllique, positif). La traduction, en supprimant l'image du voile, gomme cette dualité, un antimanichéisme systématique que développe Farah tout au long du roman. De plus, le choix de sommeil de rose, malgré toute la poésie de cette association, ne permet pas de renforcer l'image positive induite par veil d'une part, et d'autre part détruit le réseau d'intertexualité induit par cette même lexie. Enfin, il convenait de repérer que rosy sleep était une métaphore lexicalisée se traduisant par une autre métaphore lexicalisée en français, «dormir comme un bienheureux/une bienheureuse». Le choix de sommeil de rose de Surber, malgré toute sa poésie et le fait que cette réalisation renforçait subtilement la fonction épidéictique (Folkart 1999) du nom d'Ubax («fleur» en somali), introduit une difficulté d'interprétation et une version peu idiomatique. La série de choix effectuée par Surber ne permet plus ces discrets croisements et renforcements d'image. On assiste donc, dans cette traduction, à une série de microdécalages sur le plan de la saisie des référents, des contenus pragmatiques, des réseaux et même du syntagme qui aboutissent à un glissement total de sens (Folkart 1991).

And they found Idil, large as a monster and bosomy, with her large milkless breasts flat on her mound of a chest. (p. 207)

Et ils trouvèrent Idil, de la grandeur d'un monstre et plantureuse, avec ses immenses seins sans lait aplatis sur le tertre de sa poitrine. (p. 274)

Ce passage évoque le rêve éveillé de Médina, qui se voit à nouveau réunie sur une plage avec Samater, tandis qu'Ubax joue paisiblement dans l'eau non loin d'eux. 
La métaphore évoque des images très fortes, d'un côté le pouvoir et la menace (taille d'un monstre), de l'autre la mère âgée (avec ses attributs féminins à présent inutiles). Les substantifs et adjectifs choisis par Farah avaient tous une connotation négative (monster, bosomy, milkless, flat, mound of a chest) et on peut s'étonner que la traduction propose plantureuse dans ce contexte. Une poitrine plantureuse caractérise une poitrine jeune, pleine, tandis que l'adjectif est ici directement contredit par milkless et flat. Le segment final de la traduction propose par contre quelque chose d'intéressant à la fois sur le plan sémantique et phonique avec le tertre de sa poitrine (effet d'écho en $T R$ ). La valeur phonétique de cette métaphore était en effet très forte d'un bout à l'autre de la phrase avec - notamment - des rimes en ESS/EST (milkless, breast, chest) et des échos en SS. J'avancerais une autre proposition de traduction qui présente l'avantage de s'inscrire dans les thématiques de la narration et dans l'image suggérée ici tout en reconstruisant une autre phonologie (en $A / A / A$, puis en $T / T R$ ): «Apparut alors une Idil gigantesque arborant d'énormes seins taris s'étalant sur le tertre de sa poitrine.»

And they were still in the water, the two of them out in the blue deeps of the Indian Ocean, and their Ubax splashing and playful in the shallows of safety. (p. 207)

Et ils étaient encore dans l'eau, tous deux au large dans les profondeurs bleues de l'Océan Indien, et leur Ubax pataugeant et jouant dans les eaux peu profondes de la sécurité. (p. 274)

L'harmonie générale et le sentiment de paix qui se dégagent de la phrase sont renforcés par le passage métaphorique d'Ubax splashing and playful in the shallows of safety. La langue anglaise établit une distinction entre safety et security, une nuance que l'on ne retrouve pas dans le lexique français. Le problème induit par la traduction de safety (sécurité) est que cette dernière lexie est investie, dans la version anglaise de Sardines (Security Services) tout comme dans sa version française (les Services de Sécurité), d'une connotation négative: la menace des Services de Sécurité du Général qui procèdent aux purges, aux arrestations et aux tortures au beau milieu de la nuit. Les images connotées ici par safety évoquaient la protection, l'abri, la tranquillité, l'absence de danger attribuée aux eaux peu profondes de l'Océan. Il s'agissait donc de repérer à quel référent la lexie security était associée dans ce roman pour ne pas commettre d'impair. Fort de ce constat, le traducteur pouvait s'orienter sur une piste du type «dans le giron/cocon protecteur de la marée basse» pour chercher à recréer l'image globale de la phrase en proposant une métaphore qui puise dans le champ sémantique de la mer. Si «giron» et «cocon» s'inscrivent dans l'une des thématiques principales de Sardines (fertilité), l'intérêt de la deuxième option («cocon protecteur») est double: d'une part, il permettait d'éviter la métaphore lexicalisée «giron protecteur» et, d'autre part, il permettait d'inscrire la traduction dans les réseaux phonologiques développés par Farah, la métaphore originale étant en effet d'une grande richesse sur le plan prosodique: splashing/playful, shallows/safety (SPLA/PLA, SHA/SA).

Le passage ci-dessous illustre l'importance du repérage de liens inter- et intratextuels au sein de la narration.

Medina had many rooms to enter, whereas Dulman waited for only one door to open, a door into and out of which a pram could be wheeled. (p. 112)

Médina pouvait entrer par de nombreuses portes ouvertes, alors que Dulman attendait que s'ouvre une seule porte, une porte par laquelle pourrait entrer et sortir le landau qu'on y ferait rouler. (p. 152) 
Cette métaphore intervient quelques lignes plus loin que la précédente. Farah continue à explorer le thème de la stérilité, de la douleur et de l'obsession qu'elle engendre chez Dulman. Les portes représentent les possibilités d'avenir qui s'offrent à l'une et à l'autre. Médina est «gâtée» par rapport à Dulman dont les exigences se réduisent à la réalisation d'un souhait unique. La modestie de la demande se reflète dans l'adverbe only de Dulman qui s'oppose au many de Médina (abondance, faisant encore davantage écho à la fertilité) dans la proposition précédente. Il s'agissait de faire ressortir dans la traduction cette opposition entre Médina et Dulman, l'une gâtée, l'autre lésée. La traduction de Surber qui escamote l'image de la chambre vient gommer la récurrence de room dans le roman et supprime l'intratextualité et l'intertextualité dont cette lexie était investie: dès la première page de Sardines, Farah fait en effet textuellement allusion à A Room of One's Own (Virginia Woolf 1929) et, à l'instar de Woolf, lui confère une valeur très forte tout au long du roman. D'autre part, les connotations génériques dont la lexie chambre est investie soulignent encore davantage l'image du landau et du bébé tant attendu.

\section{Discussion : une traduction de la lettre peut-elle contribuer à la mise en valeur de réseaux de signifiance?}

\subsection{Vers une identification de la double fonction des métaphores chez Farah}

\section{D’après Said Samatar:}

[...] the poetry of the pastoral Somalis is didactic, with a message to convey. [...] In the scheme of Somali pastoral poetry, therefore, the doctrine of art for art's sake does not belong. [...] Given its alliterative and metrical regularity, Somali pastoral verse is easy to memorize, far more than prose. [...] Hence, Somali pastoral verse functions both as social communicator and as archival repository. (Samatar 2005:2)

Cette affirmation s'applique très justement à la double fonction des métaphores de Farah. En effet, les réseaux métaphoriques de Sardines semblent investis de deux fonctions essentielles: d'une part, illustrer indirectement («sarbeeb») ou directement les réseaux sémantiques dans la fiction de Farah et, d'autre part, frapper l'imaginaire du lecteur en favorisant leur mémorisation par l'instauration de réseaux formels et lexicaux. Samatar souligne d'ailleurs l'importance du travail exégétique que demande le décodage de certains poèmes: «hidden talk (that is, from the uninitiated and the ordinary) can only be interpreted by a poetic expert» (Samatar 2005: 4). Si les métaphores de Farah servent de prétexte à la transmission d'un message, il semble plus qu'essentiel que le traducteur se donne les clés nécessaires à leur décodage: sans travail exégétique approprié, comment reconnaître l'élément pertinent à transposer lors du processus traductif, comment déceler la présence de réseaux, tant sur le plan interthématique qu'intrathématique? Pour reprendre les concepts de Folkart, il importe d'être pleinement conscient des multiples opérations de filtrage tant sur le plan de l'encodage (lors de l'acte d'écriture) que du décodage (lors de la réception). L'étude de cas présentée plus haut révèle la présence de multiples réseaux sur lesquels repose la cohérence de la narration. Cet entrelacs peut être présenté schématiquement de la manière suivante: 


\begin{tabular}{|l|l|l|l|l|}
\hline $\begin{array}{l}\text { Réseaux transversaux } \\
\text { sous-jacents }\end{array}$ & $\begin{array}{l}\text { phonologiques } \\
\text { (dont allitératifs) }\end{array}$ & \multicolumn{2}{l|}{ lexico-sémantiques } & \multicolumn{2}{l|}{ inter- et intratextuels } \\
\hline Réseaux sémantiques: & & & & \\
\hline Pouvoir & & & & \\
\hline Liberté/épanouissement & & & & \\
\hline Plaisir & & & \\
\hline Fertilité/naissance & & & & \\
\hline
\end{tabular}

Cette représentation illustre graphiquement ce que permet, selon Berman, la mise en réseaux d'éléments signifiants sous-jacents: la mise au jour de systématismes cohérents symbolisant une dimension essentielle de l'œuvre. Cependant, la rudimentarité de ce schéma ne permet pas de mettre en lumière l'entrecroisement des réseaux sémantiques entre eux. Idéalement, un diagramme en trois dimensions aurait davantage permis de rendre compte des différentes interactions et de leur donner une perspective. Cette formule présenterait notamment l'avantage de schématiser non seulement les relations interthématiques, mais aussi intrathématiques.

\subsection{Quel projet de traduction?}

En présence de ce foisonnement de réseaux, force est de constater que la traduction mimétique utilisée par Christian Surber n'a pas sa place. On se retrouve donc confronté ici à une contradiction majeure: en affichant une traduction "fidèle» (non interventionniste), proche du texte de départ, mimétique, les décalages engendrés par la traduction n'en paraissent que plus traîtres:

[...] alors que le texte de la traduction est [...] plus homogène que celui de l'original, il est également plus incohérent, plus hétérogène et plus inconsistant. C'est un pot-pourri de divers types d'écritures. Si bien que la traduction tend toujours à apparaître comme homogène et incohérente à la fois. [...] Le lecteur perçoit [...] l'inconsistance du texte de la traduction, dans la mesure où il lui accorde rarement sa confiance [...]. (Berman 1999: 78)

Il semble que le travail heuristique mené par Surber se soit à la fois limité à la surface du texte (son épiderme), sans repérer les réseaux signifiants sous-jacents puisque ignorant la portée orale de son écriture permettant de mettre au jour ces mêmes réseaux. La voix de Farah, affublée des «vêtements d'emprunts ${ }^{3}$ » de la traduction de Surber, devient ainsi méconnaissable et perd sa mélodie globale qui constituait cependant l'une des dimensions essentielles du roman.

Isidore Okpewho, commentant les modes de traduction de la littérature orale africaine (des langues vernaculaires vers l'anglais), dénonce la tendance ayant longtemps été de mise chez les éditeurs de traductions et s'appuyant notamment sur les théories de Lévi-Strausss:

As comparative ethnologists - concerned, that is, with how the world of the "primitive" compared with that of the "civilized" man - they put almost total emphasis on units of thought and culture to the exclusion of the aesthetic substratum of the text. [...] If 
it is the message and not the medium that counts, then all that is needed is a purely functional translation - sometimes even in summary fashion - which gives us a working distillate of the text. (Okpewho 1992: 112)

La traduction mimétique ne permet pas la transposition du phénomène de réseaux vers la langue d'arrivée, parce qu'elle s'attache essentiellement à la microstructure du texte source, optant le plus souvent pour une traduction «œil pour œil, dent pour dent", alors qu'il est au contraire primordial d'inscrire sa pratique traductive dans le texte considéré comme un tout, un ensemble cohérent. La non-reconnaissance des réseaux et des rapports qui les unissent revient à nier le texte, au sens d'ensemble structuré et élaboré.

Cependant, poussées à l'extrême, les propositions d'Antoine Berman - aussi nécessaires et pertinentes qu'elles puissent être - risquent par contre d'amener le traducteur dans une impasse. En effet, si la reconnaissance des réseaux signifiants sous-jacents est essentielle lors du processus de traduction de métaphores, il appartient au traducteur, dans sa pratique traductive, de dépasser ce premier travail d'analyse afin d'établir plusieurs échelles de «signifiance». Le lexique peut ainsi avoir une importance majeure à certains moments, par exemple la lexie veil investie successivement de connotations négatives (carcan) et positives (voile protecteur), ou être mis en veilleuse au profit d'un élément formel délibérément accentué par l'auteur, tel que des considérations prosodiques (from the top of the head to the tip of her toes). Dans d'autres passages, c'est l'image obtenue, c'est-à-dire la relation même entre deux éléments du passage métaphorique qui vient soutenir l'une des thématiques majeures de la narration et qui sert de point d'ancrage dans l'extratextuel: the sun raged like an old man denied his rights, toute puissance des anciens vis-à-vis de la jeune génération en Somalie, une thématique largement développée par Farah et incarnée dans Sardines par Idil et le grand-père maternel de Médina. Si cette dernière métaphore n'est pas dépourvue d'éléments prosodiques, le traducteur devra faire un choix éclairé au vu de l'analyse préalable à la traduction. Rien ne l'empêchera, par ailleurs, de s'inscrire à un autre moment dans la pratique prosodique affichée par Farah (par ex. : proposition de retraduction avec «cocon protecteur»). À mon sens, la théorie bermanienne doit être davantage prise comme une grille d'analyse de départ, certes fondamentale sur le plan théorique, mais à appréhender avec discernement et non au pied de la lettre. En tentant de comprendre le how (Folkart 1999), et j'ajouterai même le why de la démarche de l'auteur, davantage que le what auquel semble davantage s'attacher Berman, l'auteur a plus de chances de repérer quels types de réseaux (illustrés plus haut par le schéma) réactiver lors de la traduction des métaphores. Cette série de choix peut d'ailleurs constituer ce que Berman définit comme projet de traduction: motivé, raisonné, et servant de fil conducteur à la démarche du traducteur.

\section{Conclusion : concevoir la traduction de réseaux dans un espace de dialogisme selon Bakhtine et selon Berman}

Le texte constituant par définition un réseau de significations (texte et tissu ayant la même origine étymologique), j'aimerais revenir sur le concept de dialogisme de Bakhtine, qui considère le texte comme un lieu de discussion, d'affrontement entre plusieurs voix (Todorov, 1981). Si Bakhtine entend par dialogisme l'affrontement de plusieurs voix dans une seule et même narration (discours social/sagesse populaire ou 
encore locuteur/narrateur), ce qui semble parfaitement s'appliquer à la narration de Farah, on pourrait être tenté d'élargir sa théorie en ce sens où Farah, polyglotte par nécessité et nourri de références multiculturelles par excellence, élargit dans sa pratique narrative l'éventail des voix à l'œuvre. Dans une entrevue donnée en 1994 au quotidien Libération, Farah définit d'ailleurs son monde référentiel comme schizophrène:

À la maison, nous parlions somali, langue maternelle de ce peuple colonisé parmi les colonisés. Mais nous lisions et écrivions dans d'autres langues: l'arabe, langue sacrée du Coran, l'amharique, celle de notre maître colonial pour mieux savoir ce qu'il pense, et l'anglais, langue qui pourrait un jour nous permettre de pénétrer dans un monde de signification plus vaste et laïque. Une enfance coloniale comme la mienne est par essence schizophrène [...]. (Farah 1994, cité par Morin 1997)

Cette constatation rend bien compte de la difficulté accrue du repérage des réseaux signifiants sous-jacents à l'œuvre dans Sardines: à quels univers rattacher les expressions métaphoriques à traduire? La multiplicité des ancrages culturels, malgré une prédominance indéniable de la culture somalie, confrontée à l'utilisation d'un matériau linguistique non maternel amplifie le phénomène de dialogisme évoqué par Bakhtine et repris par Kristeva et ses collaborateurs qui, dans les années 1970, ont introduit la notion d'intertextualité. En effet, les réseaux métaphoriques chez Farah représentent une cristallisation du phénomène d'intertextualité et d'intratextualité: tant sur le plan lexical, sémantique que formel. Les métaphores constituent ainsi un espace de dialogue aussi bien avec la littérature classique anglaise (Virginia Woolf: A Room of One's Own/a room in which to enter) qu'avec la littérature arabe (Les Mille et Une Nuits/and her mother had spun for her a tale of a million and one nights and spread out its fabric), le système de valeurs traditionnelles somalies (the sun raged like an old man denied his rights ne prenant de valeur que replacée dans son contexte voulant que les personnes âgées se situent en haut de l'échelle sociale et exercent un pouvoir absolu sur les jeunes générations), ou encore la prosodie et les pratiques rhétoriques de la poésie orale somalie (allitérations, phénomène de chaînage). Et si Kristeva souligne que:

[...] le texte est donc une productivité, ce qui veut dire: 1 - son rapport à la langue dans laquelle il se situe est distributif (destructivo-constructif), par conséquent, il est abordable à travers des catégories logiques plutôt que purement linguistiques; 2 - il est une permutation de textes, une intertextualité: dans l'espace d'un texte, plusieurs énoncés, pris à d'autres textes, se croisent et se neutralisent. (Julia Kristeva 1969: 52)

je pose que le traducteur a tout intérêt à penser sa démarche en inscrivant les réseaux métaphoriques dans une conception dialogique pour - à son tour - détruire et reconstruire. Le cadre théorique de l'intertextualité tel que développé par le groupe de Kristeva, permet de donner un espace de travail ouvert au traducteur puisqu'il conçoit le texte comme "un perpétuel devenir, travaillé par tous les autres textes, passés, présents et à venir (la "productivité" du texte), toujours renouvelé par le dialogisme avec d'autres textes»(Stolz 2002). Cette notion de dialogisme avec d'autres textes rejoint alors la conception bermanienne de dialogisme appliqué à la traduction - Berman entendant par là un « rapport dialogique entre langue étrangère et langue propre» (Berman 1985: 223) - offrant un cadre rêvé au traducteur, dont le texte traduit constituera, à son tour, l'un des textes futurs, perpétuant le dialogue en superposant sa voix à celles, multiples, de l'auteur. 


\section{NOTES}

1. Les «poetic chains» font partie du genre «manso», l'un des canons de la poésie somalie.

2. La lexie purdah signifie «rideau» en perse et en urdu, et correspond au hijab en arabe. Je précise que Fatima bint Thabit est d'origine perse.

3. Textuellement repris de la traduction de «borrowed garments» proposée par Christian Surber dans la version française de Sardines (p. 47).

\section{RÉFÉRENCES}

Les noms des auteurs est-africains respectent l'ordre et la forme que ceux-ci privilégient.

Abdourahman A. Waberi (1998): "Organic metaphors in two novels by Nuruddin Farah", World Literature Today, pp. 775-780.

Andrzejewski, B.W. et I. M. Lewis (1964): Somali Poetry: An Introduction, Oxford (Oxford Library of African Literature Series), Clarendon Press.

Audet, L. (2006): La création dans le processus traductif. Analyse théorique et empirique de la littérarité dans quatre traductions en français d'une nouvelle de Krúdy, thèse de doctorat, Département de linguistique et de traduction, Université de Montréal.

BavelaAr, R. (2006): “The Poetics of Displacement", Wardheernews, article en ligne du 26 juin 2006, <http://www.wardheernews.com/Articles_06/june_06/27_Poetics_Rahma.html>.

Berman, A. (1985): «La traduction comme épreuve de l'étranger», Texte, 4, pp. 67-81.

Berman, A. (1995): Pour une critique des traductions: John Donne, Paris, Éditions Gallimard, pp. 64-97.

Berman, A. (1999): «L'analytique de la traduction et la systématique de la déformation», La traduction et la lettre ou l'auberge du lointain, A. Berman (éd.), Paris, Le Seuil, pp. 49-68.

Charron, M. (2001): «Berman, étranger à lui-même?», TTR 14-2, pp. 97-121.

DANCETTE, J., L. AUDET, et L. JAY-RAYON (2007): «Axes et critères de la créativité en traduction », Meta, 52-1, pp. 108-122.

FOLKART, B. (1999): «Said Writer to Reader. Translation as Lignification», TTR, 12-2, pp. 83115.

FolKART, B. (1991): Le conflit des énonciations: traduction et discours rapporté, Candiac, Québec, Éditions Balzac.

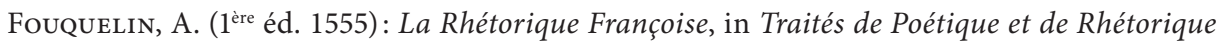
de la Renaissance, éd. F. Goyet, Paris, Le Livre de Poche nº 6720, pp. 345-464.

GiKANDI, S. (1998): «Nuruddin Farah and postcolonial textuality», Focus on Nuruddin Farah: The 1998 Neustadt Prize, World Litterature Today, 72-4, pp. 753-758.

Halverson, S. (2003): «The Cognitive Basis of Translation Universals», Target, 15-2, pp. 197241.

Henry, J. (2003): La traduction des jeux de mots, Paris, Presses Sorbonne Nouvelle.

Igboanusi, H. (2002): Igbo English in the Nigerian Novel, Ibadan, Enicrownfit Publishers.

JAY-RAYON, L. (2006): La métaphore comme traduction d'une réalité est-africaine dans le roman d'expression anglaise Sardines de l'auteur somalien Nuruddin Farah, mémoire de maîtrise, Département de linguistique et de traduction, Université de Montréal.

Johnson, J. W. (1974): Heellooy, Heelleellooy: The Development of the Genre Heello in Modern Somali Poetry, Bloomington, Indiana University Press, pp. 36-37.

Kintsch, W. (1998): Comprehension. A Paradigm for Cognition, Cambridge/New York, Cambridge University Press.

KristeVA, J. (1969): Semeiotikè, recherches pour une sémanalyse, Paris, Le Seuil.

Lakoff, G. (1987): Women, Fire and other Dangerous Things: What Categories Reveal about the Mind, Chicago, University of Chicago Press.

Lakoff, G. and M. Johnson (1980): Metaphors We Live by, Chicago/London, The University of Chicago Press.

Lakoff, G. and M. Turner (1989): More than Cool Reason: A Field Guide to Poetic Metaphor, Chicago, The University of Chicago Press. 
Molinié, G. (1993): La stylistique, Paris, PUF, (Collection Premier Cycle).

Morin, D. (1997) : «Littérature et politique en Somalie», CNRS, Travaux et documents du CEAN $\mathrm{n}^{\circ} 56$.

Ngũgĩ, W. T. (1986): Decolonising the Mind: The Politics of Language in African Literature, London, James Currey, Nairobi, Heinemann Kenya/New Hampshire, Heinemann.

Nuruddin, M. F. (1992) [@1980] : Sardines, Saint-Paul, Graywolf Press.

Nuruddin, M. F. (1995): Sardines, traduit par Christian Surber, Carouge-Genève, Zoé.

Nuruddin, M. F. (1994): entrevue publiée dans Libération du 17 novembre 1994 (citée par Didier Morin).

Окрешно, I. (1992): African Oral Literature: Backgrounds, Character, and Continuity, Bloomington, Indiana University Press.

Prandi, M. (2000): «Littéral, non littéral, figuré», Cahiers de praxématique, 35, Montpellier, Éditions de l'Université Paul Valéry.

Rushiy, K. (2005): "Unbroken Chain”, The Guardian, article en ligne du 15 octobre 2005, $<$ http://books.guardian.co.uk/review/story/0,12084,1591589,00.html\#article_continue $>$.

Rushdie, S. (1992) [@1982] : Imaginary Homelands, London, Granta Books.

Rydning, A. F. (2004): «Le défi du procédé synecdoquien en traduction», Meta, 49-4, pp. 856875.

Samatar, S. (2005): «Sarbeeb: The Art of Oblique Communication in somali Culture», Wardheernews, article en ligne du 9 juin 2005, <http://www.wardheernews.com/articles/ june/09_Sarbeeb_Samatar.htm>.

Stolz, C. (2002): «Polyphonie et intertextualité», article en ligne affiché le site de Fabula, la recherche en littérature: $<$ http://www.fabula.org/atelier.php?action=browse\&id=Polyphon ie_et_intertextualit\%26eacute\%3B\&imprimer $=1>$.

TAmpa-Besz, I. (1981): Le sens figuré, Paris, PUF.

Todorov, T. (1981): Mikhail Bakhtine, le principe dialogique, Paris, Le Seuil.

VANDAELE, S. (2002): «Métaphores conceptuelles en traduction biomédicale et cohérence», TTR, 15-1, pp. 223-240.

Woolf, V. (1963) [@1928] : A Room of One’s Own, Harmondsworth, Middlesex, Penguin. 